\title{
Hybrid H-mode scenario with nitrogen seeding and type III ELMs in JET
}

\author{
Y Corre $^{1}$, E Joffrin ${ }^{1}$, P Monier-Garbet ${ }^{1}$, Y Andrew ${ }^{2}$, G Arnoux ${ }^{1}$, \\ M Beurskens ${ }^{2}$, S Brezinsek ${ }^{3}$, M Brix ${ }^{2}$, R Buttery ${ }^{2}$, I Coffey ${ }^{4}$, K Crombe $^{5}$, \\ E de La Luna ${ }^{6}$, R Felton ${ }^{2}$, C Giroud ${ }^{2}$, S Hacquin ${ }^{1}$, J Hobirk ${ }^{7}$, A Huber ${ }^{3}$, \\ F Imbeaux ${ }^{1}$, S Jachmich ${ }^{8}$, M Kempenaars ${ }^{2}$, X Litaudon ${ }^{1}$, H Leggate ${ }^{2}$, \\ T Loarer ${ }^{1}$, G Maddison ${ }^{2}$, E Rachlew ${ }^{9}$, J Rapp ${ }^{3,10}$, O Sauter ${ }^{11}$, \\ A Savchkov $^{2}$, G Telesca ${ }^{3}$, A Widdowson ${ }^{3}$, K D Zastrow ${ }^{2}$, \\ O Zimmermann ${ }^{3}$ and JET-EFDA collaborators ${ }^{12}$
}

${ }^{1}$ CEA, IRFM, F-13108 Saint-Paul-lez-Durance, France

2 EURATOM/UKAEA Association, Culham Science Centre, Abingdon OX14 3DB, UK

${ }^{3}$ Institut für Energieforschung-Plasmaphysik, Forschungszentrum Jülich, Association EURATOM-FZJ Trilateral Euregio Cluster, Germany

${ }^{4}$ Queen's University, Belfast, BT7 1NN, UK

5 Department of Applied Physics, Ghent University, Rozier 44, B-9000 Ghent, Belgium

${ }^{6}$ EURATOM-CIEMAT, Laboratorio National de Fusion, Spain

${ }^{7}$ Max-Planck-Institut für Plasmaphysik, EURATOM Association, Garching, Germany

${ }^{8}$ Association EURATOM-Belgian State, KMS-ERM B-1000, Brussels, Belgium

${ }^{9}$ Association EURATOM-VR, KTH Royal Institute of Technology, SE-10691 Stockholm, Sweden

${ }^{10}$ FOM Instituut voor Plasmafysica Rijnhuizen, EURATOM Association, Trilateral Euregio Cluster, Nieuwegein, The Netherlands

${ }^{11}$ Association EURATOM Confédération Suisse, CRPP_EPFL, 1015 Lausanne, Switzerland

E-mail: yann.corre@cea.fr

Received 16 June 2008, in final form 4 September 2008

Published 15 October 2008

Online at stacks.iop.org/PPCF/50/115012

\begin{abstract}
The performance of the 'hybrid' H-mode regime (long pulse operation with high neutron fluency) has been extensively investigated in JET during the 2005-2007 experimental campaign up to normalized pressure $\beta_{\mathrm{N}}=3$, toroidal magnetic field $B_{\mathrm{t}}=1.7 \mathrm{~T}$, with type I ELMs plasma edge conditions. The optimized external current drive sources, self-generated non-inductive bootstrap current and plasma core stability properties provide a good prospect of achieving a high fusion gain at reduced plasma current for long durations in ITER.

One of the remaining issues is the erosion of the divertor target plates associated with the type I ELM regime. A possible solution could be to operate with a plasma edge in the type III ELM regime (reduced transient and stationary heat loads) obtained with impurity seeding. An integrated hybrid type III ELM
\end{abstract}

\footnotetext{
12 See the appendix of M L Watkins et al 2006 Proc. 21st Int. Conf. on Fusion Energy 2006 (Chengdu, China) (Vienna: IAEA).
} 
regime with a normalized pressure $\beta_{\mathrm{N}}=2.6\left(P_{\mathrm{NBI}} \sim 20-22 \mathrm{MW}\right)$ and a thermal confinement factor of $\mathrm{H}_{98}^{*}(y, 2) \sim 0.83$ has been recently successfully developed on JET with nitrogen seeding. This scenario shows good plasma edge condition (compatible with the future ITER-like wall on JET) and moderate MHD activity. In this paper, we report on the experimental development of the scenario (with plasma current $I_{\mathrm{p}}=1.7 \mathrm{MA}$ and magnetic field $B_{\mathrm{t}}=1.7 \mathrm{~T}$ ) and the trade-off between heat load reduction at the target plates and global confinement due to nitrogen seeding and type III ELM working conditions.

(Some figures in this article are in colour only in the electronic version)

\section{Introduction}

The reference H-mode scenario ( $\mathrm{H}$ for high confinement) for ITER [1] is expected to meet the goal of fusion gain $Q=10$ at $\beta_{\mathrm{N}}=1.8$ for a few hundred seconds. To increase discharge duration in ITER with sufficient fusion gain $(Q>5)$, it is necessary to maximize the selfgenerated non-inductive current fraction by decreasing the plasma current and increasing the plasma pressure. Higher pressure could generate pressure driven plasma instability such as the neo-classical tearing modes NTMs that are often triggered by sawtooth collapse. In this context, the so-called 'hybrid' scenario has successfully eliminated the deleterious effect of sawteeth activity by establishing a broad current density profile in stationary state with safety factor $q$ close to unity in the plasma core. Originally operated in ASDEX Upgrade [2] and DIII-D [3], extended in JET [4], this scenario provides a promising route to ITER operation with reduced flux consumption and high neutron fluence [5]. Similarly to the standard H-mode, two of the remaining issues of the 'hybrid' scenario for long pulse operation with the type I ELM regime are that of first wall material erosion (which limits the lifetime of plasma facing componentsPFCs) and of heat loads which can lead to unacceptable damage of PFCs (melting, sublimation, cracks, boiling of the water in the cooling pipes and ultimately water leaks).

Due to the problem of tritium (T) retention in carbon PFCs, the materials presently envisaged in ITER are beryllium (Be) in the main chamber, tungsten (W) in the divertor baffle region and carbon (C) for a restricted number of targets (at the location with the highest heat load) to minimize T-retention in the vessel. To test ITER-relevant plasma scenarios in the JET tokamak, it is planned to install a beryllium first wall and apply a tungsten coating [6] on the full set of tiles in the divertor (ITER-like wall project [7]). In ITER (actively cooled device), but also on JET with the future ITER-like wall (inertial cooling device), low erosion rates and sustainable heat loads (transient and stationary) will be necessary to preserve the integrity of the machines. In order to prepare the operation of 'hybrid' discharges with the ITER-like wall in JET and long 'hybrid' discharges in ITER, we have investigated the capability of impurity seeding to reduce the heat loads and the feasibility to operate the 'hybrid' scenario with type III ELM [8] (where mitigation of both transient and stationary heat loads is possible in contrast to type I ELM regime where only stationary heat load mitigation has been achieved [9]).

During ELMy H-mode operation, when external gas fuelling is applied, the ELM frequency increases while the energy confinement is reduced; further increase in the gas fuelling usually leads to the transition from type I to type III ELM [10]. Type III ELMs are likely to be caused by resistive ballooning instabilities [11], thus favorably at high plasma collisionality $\left(v_{\mathrm{e}}^{*}=Z_{\mathrm{eff}} n_{\mathrm{e}} q R / T_{\mathrm{e}}^{2}\right)$. According to a wide experimental study dedicated to the standard 
H-mode scenario on JET, it turns out that at the transition from type I to type III ELM, the pedestal temperature (and pressure) always drops off whether the pedestal density increases or decreases [12]. Impurity seeding is used in our experiment to modify the edge plasma parameter in that sense (reduced pedestal temperature). Nitrogen impurity is chosen here because of its high radiating efficiency at low plasma temperature. In JET attached plasmas, nitrogen (ionization potential of $E_{\mathrm{i}}=15.58 \mathrm{eV}$ ) is expected to radiate mainly in the divertor near the X-point and upstream of the divertor targets. Impurity seeding has initially been applied to standard H-mode discharges to achieve type III ELMs. After successful application [8], impurity seeding is extended to the 'hybrid' H-mode regime initially performed with type I ELMs (with plasma current $I_{\mathrm{p}}=1.7 \mathrm{MA}$ and toroidal magnetic field $B_{\mathrm{t}}=1.7 \mathrm{~T}$ ). One of the main issues of such an experiment was to establish if the hybrid scenario (characterized here by the MHD stability rather than the energy confinement) could be sustained with type III ELMy H-mode in the plasma edge. In the reference 'hybrid' regime, the maximum radiated power fraction achieved with deuterium fuelling alone is $P^{\text {rad }} / P^{\text {tot }} \sim 35 \%$ (mainly C plus substantial $\mathrm{D}$ radiation). An additional nitrogen injection brings the radiated fraction up to $50 \%$ (mainly C plus $\mathrm{N}$ radiation). This corresponds to the type III ELM regime (obtained here when the pedestal temperature $T^{\text {ped }} \leqslant 750 \mathrm{eV}$ and $P^{\text {rad }} / P^{\text {tot }}>40 \%$ ).

The set-up and the description of the hybrid scenario with impurity seeding are presented in section 2 . The plasma modifications due to $\mathrm{N}$ seeding are discussed separately during the type I ELM regime (section 3) and type III ELM regime (section 4). A comparison between D and $\mathrm{N}$ injection is also proposed in section 3 in order to balance the advantages and disadvantages regarding heat loads and plasma confinement. Analysis of the plasma characteristics (radiation, temperature, density and heat loads) into the pedestal region, near the X-point, into the scrapeoff layer and near the target plates, respectively, is presented. A net reduction of the transient (during ELM) and stationary (inter-ELM) heat loads on the divertor tiles is achieved during the type III ELM regime at the expense of the plasma energy confinement. The trade-off between heat load reduction and plasma performance degradation due to impurity seeding is discussed in section 5 and conclusions are drawn in section 6.

\section{Description of the hybrid H-mode scenario with impurity seeding}

\subsection{Hybrid scenario experimental set-up}

The target plasma scenario is a hybrid H-mode (defined here as an optimized scenario for high $\beta_{\mathrm{N}}$ operation with moderate MHD activity) with type I ELMs (\#68505), where pedestal plasma temperature is $T^{\text {ped }} \sim 1000 \mathrm{eV}$, plasma current $I_{\mathrm{p}}=1.7 \mathrm{MA}$, toroidal magnetic field $B_{\mathrm{t}}=1.7 \mathrm{~T}$, central density $\left\langle n_{\mathrm{e}}\right\rangle \sim 5 \times 10^{19} \mathrm{~m}^{-3}$ ( $\sim 70 \%$ of the Greenwald density) and edge safety factor $q_{95} \sim 3.2$ in which the injected neutral beam power (NBI) is feedback controlled to $\sim 14-16 \mathrm{MW}$ to achieve a total normalized pressure of $\beta_{\mathrm{N}}=3$. The thermal confinement enhancement factor achieved in the target plasma scenario is $\mathrm{H}_{98}^{*}(y, 2) \sim 1.05$ and the plasma effective charge is $Z_{\text {eff }} \sim 1.8$. A high triangularity magnetic configuration $(\delta=0.44)$ is used with a full carbon MkII-HD divertor (with a load-bearing septum replacement plate (LBSRP) as shown in figure 1). Lower hybrid heating is used during the plasma current ramp-up (for a duration of $\sim 3 \mathrm{~s}$ ) to delay the plasma current profile penetration with the aim of producing a broad $q$-profile when the main heating is applied. This is followed by an intermediate $\beta_{\mathrm{N}}=2$ phase (for a duration of $\sim 3 \mathrm{~s}$ ) for stabilization of the $q$-profile close to 1 in order to minimize the impact of sawtooth on stability. The $\beta_{\mathrm{N}}$ request is then increased and kept constant during $4 \mathrm{~s}$. During this phase, a preset injection of deuterium is applied: $\beta_{\mathrm{N}} \sim 3$ has been obtained 


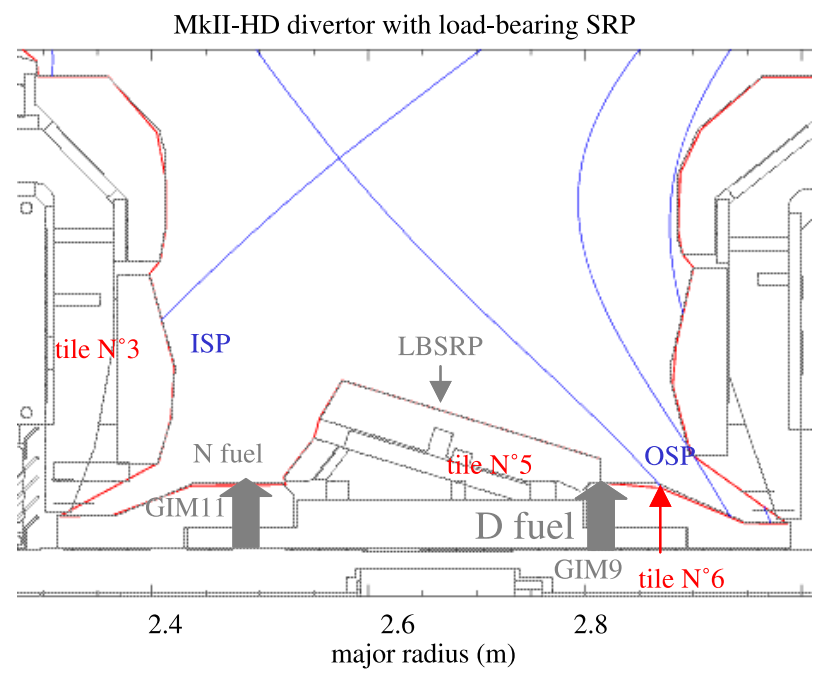

Figure 1. High triangularity magnetic configuration $(\delta=0.44)$ and schematic of the MkII$\mathrm{HD}$ divertor with load-bearing septum replacement plate (LBSRP). The $\mathrm{N}_{2}$ and $\mathrm{D}_{2}$ gas injection modules are located on the inner and outer sides, respectively. The last close surface shows the $\mathrm{X}$-point and indicates the inner and outer strike points (ISP and OSP).

with low deuterium fuelling (hybrid reference no $1 \# 68505$ : $\Phi_{\mathrm{D}}=0.6 \times 10^{22}$ electrons per second) and $\beta_{\mathrm{N}}=2.6$ with high deuterium fuelling and density close to the Greenwald density $n_{\mathrm{e}} \sim 0.95 \cdot n_{\mathrm{Gr}}$ (hybrid reference no $2 \# 68515,68738: \Phi_{\mathrm{D}}=5 \times 10^{22} \mathrm{e} \mathrm{s}^{-1}$ ).

\subsection{Seeding technique on the scenario}

Nitrogen injection is applied in addition to deuterium fuelling during the first three seconds of the high $\beta_{\mathrm{N}}$ plateau (when $\beta_{\mathrm{N}}=2.6$ ). Deuterium is injected in the bottom of the divertor near the outer strike point (OSP) on the low-field side (LFS) while nitrogen is injected into the private-flux region from the horizontal target plate located on the high-field side (HFS), as shown in figure 1.

The experimental scenario and timing are presented in figure 2 with pre-programmed nitrogen injection (preset fuelling rate). A real-time control feedback technique can also be applied to control the radiated power (measured with the bolometric system) with the nitrogen injection during the high- $\beta_{\mathrm{N}}$ plateau. The feedback controller includes the filtering of the radiation fraction and uses both integral and derivative gains as described in [13]. The realtime control feedback is an efficient tool to find the quantity of gas needed to reach high radiative fraction and thus find the transition from type I to type III ELM regime.

\subsection{Operational domain covered during the experiment}

The maximum radiated power fraction achieved with deuterium fuelling alone (with mainly $\mathrm{D}$ and $\mathrm{C}$ radiators) is $P^{\mathrm{rad}} / P^{\text {tot }} \sim 37 \%$ with density close to the Greenwald limit $n_{\mathrm{e}} \sim n_{\mathrm{Gr}}$ (\#68739). Figure 3(a) shows the limit of the radiated fraction achieved with deuterium fuelling alone (when the density approaches the Greenwald limit, the fuelling rate increases exponentially while the radiated fraction stays quasi-constant). Using deuterium plus nitrogen 


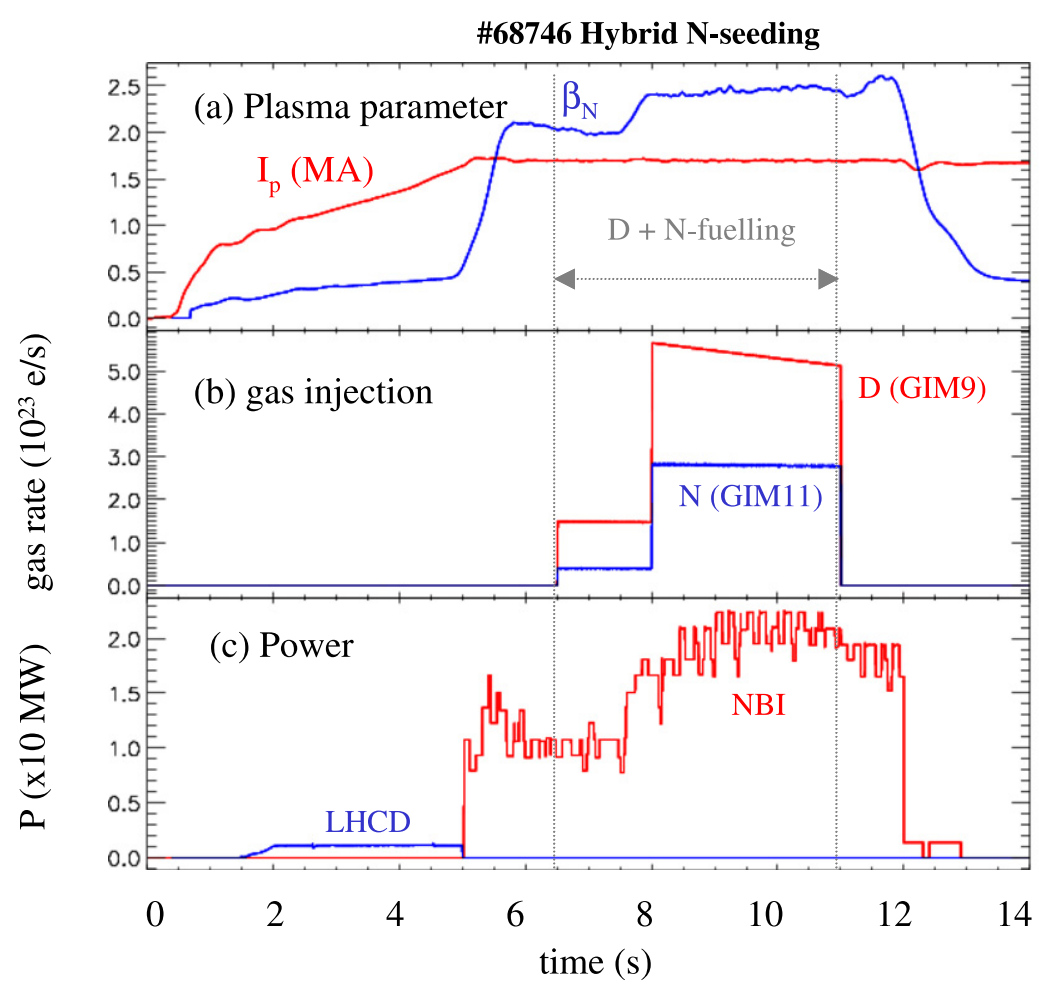

Figure 2. Plasma parameters and timing used to develop the hybrid type III ELM scenario. Shot \#68746 with pre-programmed $\mathrm{N}$ injection. (a) plasma current $I_{\mathrm{p}}$ and total normalized pressure $\beta_{\mathrm{N}}$, (b) sub-divertor deuterium and nitrogen fuelling (given in electrons per second); gas introduction modules are presented in figure $1,(c)$ neutral beam and lower hybrid power.

fuelling enables to increase the radiative fraction (with mainly $\mathrm{D}, \mathrm{C}$ and $\mathrm{N}$ radiators); $50 \%$ has been achieved during the experiment (\#68532 in figure 3(a)). These values have been determined by using an average of the bolometer signal (sampling rate of $2 \mathrm{~ms}$ ) during the interELM period. Since the radiation during ELMs is higher (peaks of radiation) this corresponds basically to the baseline of the bolometer time trace. The type III ELM regime, characterized by high ELM frequency $(\sim 500 \mathrm{~Hz})$ and small amplitude (visible and IR transient peaks are reduced), is achieved here when $P^{\mathrm{rad}} / P^{\text {tot }}>40 \%$ and the pedestal ion temperature $T^{\text {ped }}$ is below $750 \mathrm{eV}$. Figures 3(a) (fuelling and radiation) and (b) (pedestal temperature and density) summarized the operational domain achieved to develop the hybrid type III ELM regime in JET (including pre-programmed and feedback seeding discharges). A particular and intermediate regime characterized by the occurrence of compound ELMs (i.e. mixed type I/type III ELMs separated by brief ELM free periods) is obtained for high pedestal densities, when approaching the type III ELM plasma conditions (as shown in figure 3(b)). The distinction between the compounds ELM regime with the standard type I ELM regime is sometimes very narrow and thus difficult to establish correctly without any optimized diagnostic set-up and power variation (traditionally used to make the distinction). Discharges including compound ELMs are reported in figure $3(b)$ within the dashed circle.

Figure 4 shows some of the main plasma parameters for the hybrid type III ELM regime (\#68532 obtained with both radiative and $\beta_{\mathrm{N}}$ real-time control) compared with the reference hybrid type I ELM regime with high D fuelling (\#68515 obtained with $\beta_{\mathrm{N}}$ real-time 

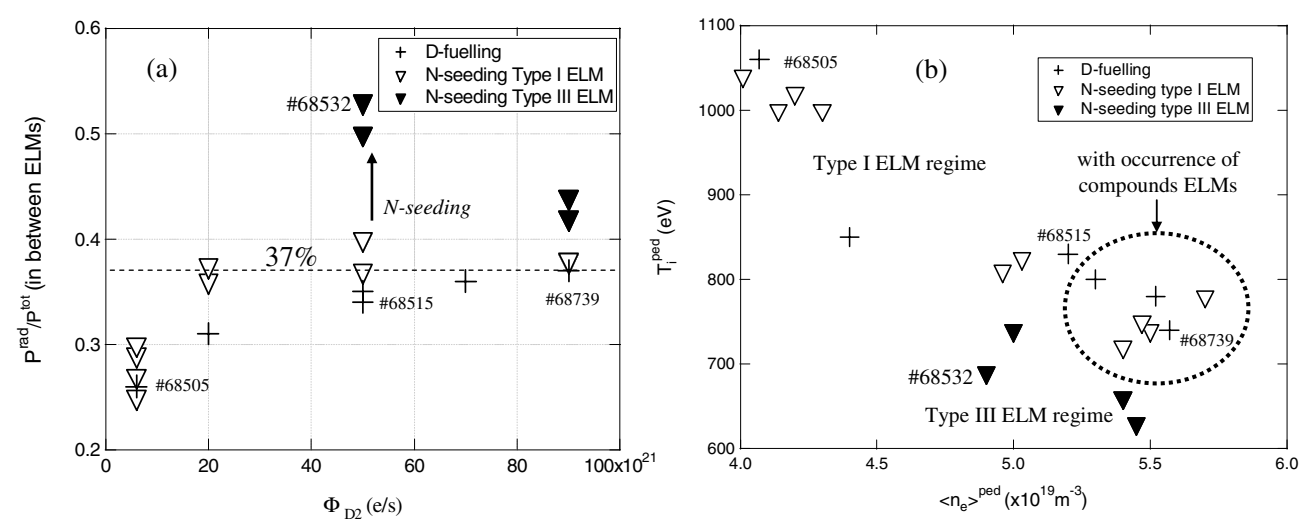

Figure 3. Set of JET discharges with various $\mathrm{N}_{2}$ (HFS) and $\mathrm{D}_{2}$ (LFS) fuelling rate. (a) Radiated fraction measured with the bolometers as a function of the deuterium fuelling rate (given in electron per second - the particle influx is obtained by using the atomic number and the charge factor, $Z=14$ for $\mathrm{N}_{2}$ and $Z=2$ for $\mathrm{D}_{2}$ ). (b) Operational domain achieved with type I ELM and type III ELM regimes: $T_{\mathrm{i}}^{\text {ped }}$ measured with the edge charge exchange recombination spectroscopy (CXRS) diagnostic (averaged near the top of the pedestal) as a function of $\left\langle n_{\mathrm{e}}^{\text {ped }}\right\rangle$ estimated with the interferometer diagnostic (vertical line average): Crosses represent discharges with D injection. Triangles represent discharges with D and N injection. Discharges where the type III ELM regime is achieved are filled up with black and type I ELM regime are filled up with white (including discharges with compounds ELMs).
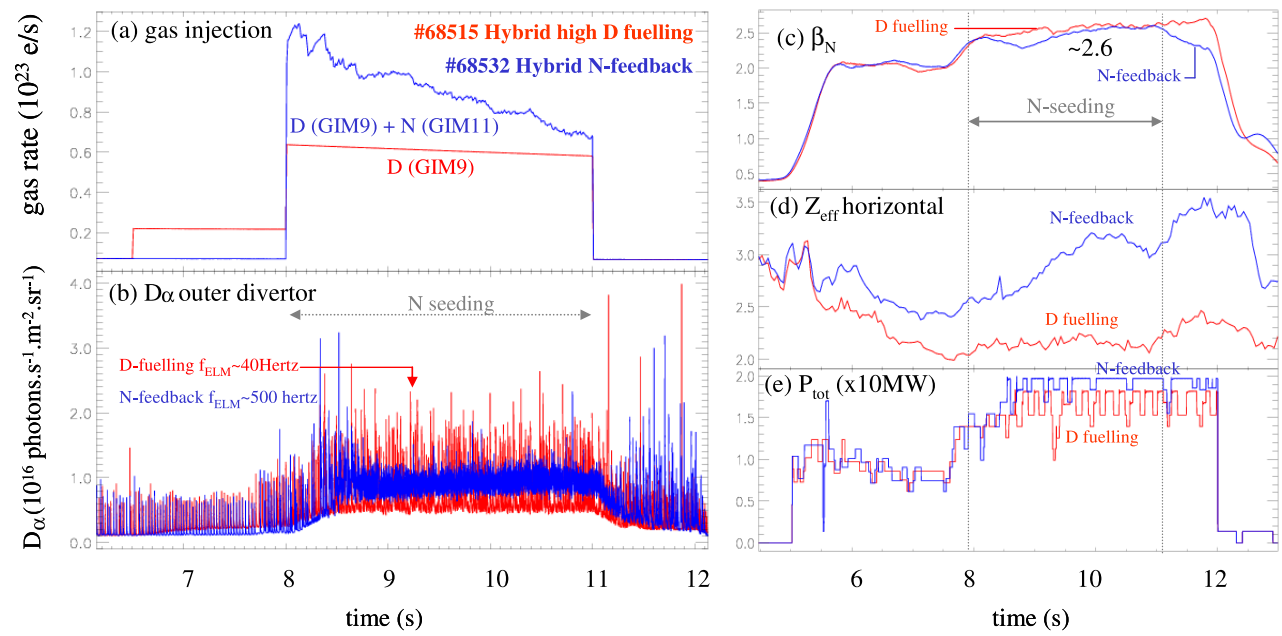

Figure 4. (a) Sub-divertor gas fuelling; gas introduction modules are presented in figure 1, (b) $D_{\alpha}$ signal in the outer divertor, $(c)$ total normalized pressure $\beta_{\mathrm{N}},(d)$ effective charge $Z_{\text {eff }}$ estimated with the visible spectroscopy (chord in equatorial plan) and $(e)$ neutral beam power for the reference type I (D fuelling) and type III (N-feedback) ELM regimes.

control only). In the seeded discharge, the strong initial gas puff $\left(\Phi_{\mathrm{D}}=5 \times 10^{22} \mathrm{e} \mathrm{s}^{-1}\right.$ and $\Phi_{\mathrm{N}} \sim 7 \times 10^{22} \mathrm{e} \mathrm{s}^{-1}$ ) decreases the pedestal temperature (from $\sim 1000 \mathrm{eV}$ to $\sim 700 \mathrm{eV}$ ) and produces the transition to the type III ELM regime.

Before the transition phase, i.e. during the type I ELM regime (when $P^{\mathrm{rad}} / P^{\text {tot }}<40 \%$ and $T^{\text {ped }}>750 \mathrm{eV}$ ), a significant leakage of the seeded impurity out of the divertor is observed, 
(a) q-profile

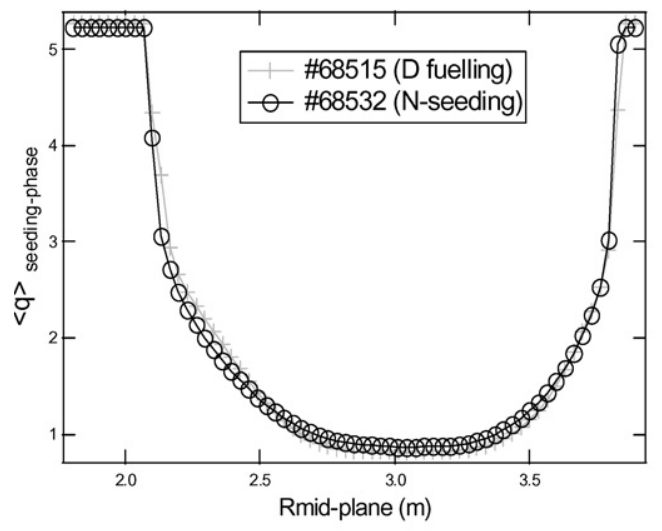

(b) MHD activity \#68532

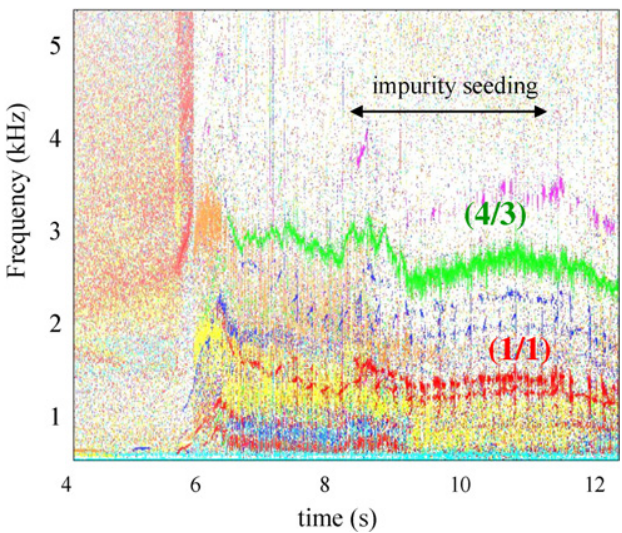

Figure 5. (a) Time average of $q$-profiles measured by the MSE system during the seeded phase $t=[8-11 \mathrm{~s}]$ for the pulse with D fuelling only (crosses) and $\mathrm{D}+\mathrm{N}$ fuelling (circles). (b) Spectrogram of one pick-up magnetic coil signal showing the MHD activity during shot \#68532.

as also reported in [14]. Once the pedestal temperature is reduced and the type III ELM is obtained, the impurity gas puff needed to maintain constant radiated power and therefore the regime stationary decreases slowly from $\Phi_{\mathrm{N}} \sim 6 \times 10^{22}$ down to $\Phi_{\mathrm{N}} \sim 3 \times 10^{22} \mathrm{e} \mathrm{s}^{-1}$. The total radiated power fraction achieved with the type III ELM regime is $\sim 50 \%$ with $\beta_{\mathrm{N}}=2.6$ $\left(P_{\mathrm{NBI}} \sim 20-22 \mathrm{MW}\right)$ and the thermal confinement enhancement factor is $\mathrm{H}_{98}^{*}(y, 2) \sim 0.83$ (\#68532), about 20\% less than the reference type I ELM discharge (\#68505). The confinement loss is due to the pedestal energy degradation and the residual MHD activity (see the next section). The positive effect associated with the type III ELM regime is a net reduction of the heat load on the divertor tiles that are normally subject to high heat flux (discussed in details in section 4). Also discussed in section 4 is the nitrogen impurity concentration responsible for the relatively high $Z_{\text {eff }}$ observed in the hybrid type III ELM (figure $4(d)$ ). Before $\mathrm{N}$ seeding $(t<8 \mathrm{~s})$, the difference in $Z_{\text {eff }}$ is due to trapped and released nitrogen from preceding discharges to \#68532. During $N$ seeding $(t=8-11 \mathrm{~s}), Z_{\text {eff }}$ increases and stabilizes around 3. The plasma modification due to $\mathrm{N}_{2}$ injection in the type I ELM regime is discussed separately in section 3 .

\subsection{MHD and plasma stability}

The MHD activity is characterized by mild $n=1$ sawteeth precursors present during the high $\beta_{\mathrm{N}}$ phase (visible by the burst in the magnetic spectrogram depicted in figure $5(b)$ ), suggesting that the minimum safety factor $q_{\mathrm{min}}$ is equal to or smaller than 1 (as confirmed with the motional Stark effect measurement presented in figure $5(a)$ ). During the hybrid type III ELM, an $n=3$ mode (destabilized at $t=6 \mathrm{~s}$ before the impurity seeding is applied, i.e. when $\beta_{\mathrm{N}}=2$ ) is present near the $q=4 / 3$ surface, as described in [15]. The more deleterious $2 / 1$ and $3 / 2$ NTMs are not present during the hybrid type III ELM case or in the reference type I case. The residual MHD island (4/3 NTM) is about $3 \mathrm{~cm}$ wide and explains only partially the confinement degradation observed during the type III ELM regime (compared with the type I ELM case in which the 4/3 NTM is not present): the loss of confinement associated with the 4/3 NTM is estimated to be $\delta H=4 \cdot($ width $/ a) \cdot\left(\rho_{4 / 3} / a\right)^{3} \approx 5 \%$, where $a$ is the minor plasma radius and $\rho_{4 / 3}$ is the radial position of the $q=4 / 3$ magnetic surface [16]. 
Table 1. Summary of the D- and N-fuelling scans: fuelling rate $\left(\mathrm{e} \mathrm{s}^{-1}\right)$, averaged ELM frequency (Hz), ELM type and averaged total injected power (MW). The last two columns show discharges discussed later on in the paper (see section 5)_\#68532 is the reference hybrid type-III ELM.

\begin{tabular}{|c|c|c|c|c|c|c|c|c|c|c|c|}
\hline \# & 68505 & 68507 & 68508 & 68509 & 68510 & 68514 & 68515 & 68516 & 68739 & 68746 & 68532 \\
\hline$\Phi_{\mathrm{D}}$ & $0.6 \mathrm{e} 22$ & $0.6 \mathrm{e} 22$ & $0.6 \mathrm{e} 22$ & $0.6 \mathrm{e} 22$ & $0.6 \mathrm{e} 22$ & $2 \mathrm{e} 22$ & $5 e 22$ & $7 \mathrm{e} 22$ & $9 \mathrm{e} 22$ & $5 e 22$ & $5 e 22$ \\
\hline$\Phi_{\mathrm{N}}$ & 0 & $2 \mathrm{e} 21$ & $7.5 \mathrm{e} 21$ & $1.6 \mathrm{e} 22$ & $2.1 \mathrm{e} 22$ & 0 & 0 & 0 & 0 & $2.7 \mathrm{e} 21$ & FB \\
\hline$\left\langle f_{\mathrm{ELM}}\right\rangle$ & 50 & 50 & 50 & 40 & 40 & 65 & 60 & 60 & 65 & $>100$ & 500 \\
\hline type & I & I & I & I & I & I & I & I & Mix & Mix & III \\
\hline$\left\langle P^{\mathrm{tot}}\right\rangle$ & 15 & 15.5 & 15.5 & 16 & 16 & 16.5 & 17 & 18 & 19 & 19.5 & 19.5 \\
\hline
\end{tabular}

\section{Plasma modification due to $\mathrm{D}$ and $\mathrm{N}$ fuelling during the hybrid type I ELM regime}

\subsection{Effect of fuelling on pedestal}

To assess separately the effect of $\mathrm{D}$ fuelling and $\mathrm{N}$ seeding on the plasma edge characteristics, a series of D and $\mathrm{N}$ pre-programmed fuelling scans have been performed in the reference hybrid type I ELM scenario. The D- and N-fuelling values designed for the scans are reported in the number of electrons per second $\left(\mathrm{e} \mathrm{s}^{-1}\right)$. The D-fuelling scan spans from low to high electron fuelling rates $\Phi_{\mathrm{D}}=(0.6-9) \times 10^{22} \mathrm{e} \mathrm{s}^{-1}$ (the $\mathrm{D}_{2}$ influx is equivalent to the number of electrons injected per second divided by the atomic number $N=2$ ), corresponding to the following series of discharges: \#68505, \#68515, \#68516 and \#68739 (see table 1). The highest value of the D-fuelling rate scheduled in the experiment was limited by the energy confinement drop (the thermal confinement factor measured during \#68739 is $\mathrm{H}_{98}^{*}(y, 2) \sim 0.82$ ) and because $P^{\text {rad }} / P^{\text {tot }}$ does not increase anymore (as shown in figure $3(a)$ ). The $\mathrm{N}$-fuelling scan is limited to low electron fuelling rates in the $\Phi_{\mathrm{N}}=(0.6-2.1) \times 10^{22} \mathrm{e} \mathrm{s}^{-1}$ range (the $\mathrm{N}_{2}$ influx is obtained by dividing the electron flux by the charge factor $Z=7$ and the atomic number $N=2$ ); this corresponds to the following series of discharges: \#68505, \#68507, \#68508, \#68509 and \#68510 (see table 1). The highest value of the $\mathrm{N}$-fuelling rate scheduled in the experiment was limited by the need to keep the effective charge of the plasma below a value of $Z_{\text {eff }} \sim 3$. The modification of the plasma ion temperature (measured by the edge CXRS diagnostic [17]) due to $\mathrm{D}$ and $\mathrm{N}$ fuelling is presented in figure 6.

Increasing deuterium fuelling reduces the ion temperature at the top of the pedestal from $1000 \mathrm{eV}$ to below $800 \mathrm{eV}$, while the electron density at the pedestal varies from $\left\langle n_{\mathrm{e}}^{\text {ped }}\right\rangle \sim$ $4 \times 10^{19} \mathrm{~m}^{-3}$ to $\sim 5.5 \times 10^{19} \mathrm{~m}^{-3}$ and the radiated fraction increases from $P^{\text {rad }} / P^{\text {tot }} \sim 25 \%$ to $\sim 35 \%$. On the other hand, at low plasma density and with the limited level of $\mathrm{N}$-fuelling rates used in the experiment, increasing nitrogen fuelling does not increase the radiation significantly and consequently does not influence the plasma ion temperatures (circles in figure $6(a)$ ) while $Z_{\text {eff }}$ is varying from $\sim 1.8$ (\#68505) to $\sim 2.7$ (\#68510, a value that sets a limit on the $\mathrm{N}$ injection when the D fuelling is low). The cooling effect observed in the pedestal region with D injection is coherent with the increase in density and radiated power (also correlated with the occurrence of compound ELMs), as shown in figure $6(b)$ ( $P^{\mathrm{rad}}$ increases from 4.5 to 7.5 MW). The experimental strategy used to develop the hybrid type III ELM scenario is to cool the pedestal with deuterium and nitrogen injection in order to optimize, simultaneously, the radiation efficiency (to access the type III ELM regime) and $Z_{\mathrm{eff}}$.

\subsection{Effect of fuelling on heat load}

The effect of $\mathrm{D}$ and $\mathrm{N}$ fuelling on the heat load is different from that observed on the ion temperature. It has been established in [9] that the transient heat load in the type I ELM regime 

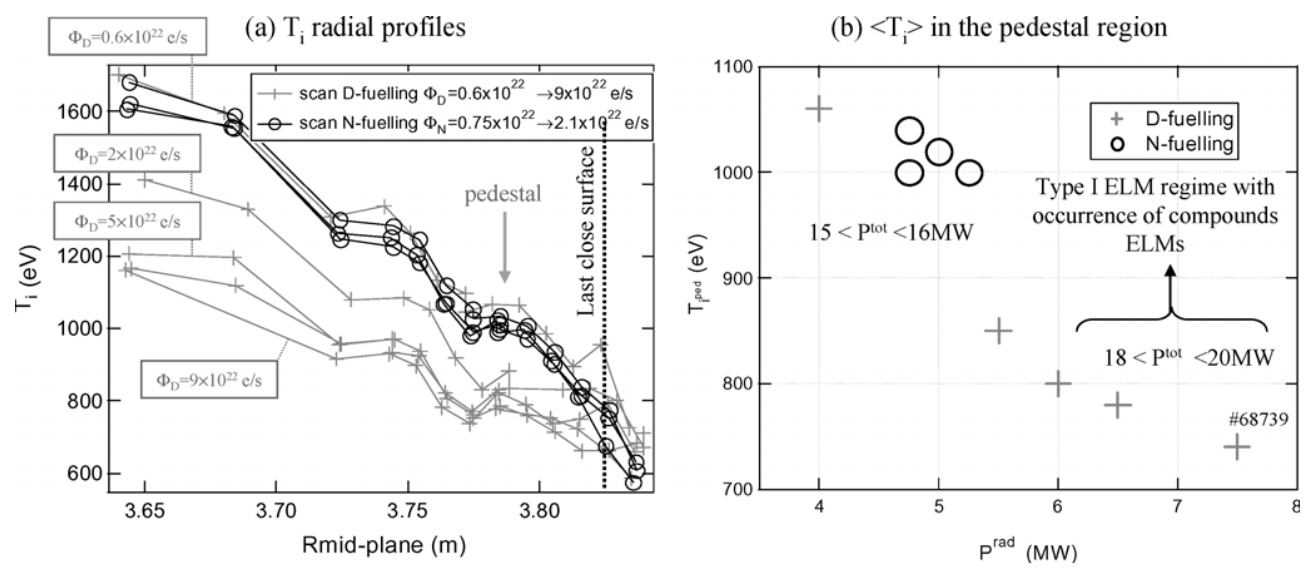

Figure 6. Ion temperature measured with the edge CXRS diagnostic. (a) $T_{\text {i }}$ profiles as a function of the mid-plane position for a series of pulses with $\mathrm{D}$ (crosses) and $\mathrm{N}$ (circles) pre-programmed injection (equivalent electron influx). (b) Time average of $T_{\mathrm{i}}$ in the pedestal region as a function of the radiated power for the same series of pulses.

is only marginally affected by impurity radiation (ELM mitigation with impurity seeding does not appear to be an efficient tool in JET ELMy H-mode plasmas). In this section (dedicated to the type I ELM regime), we focus therefore our interest on the stationary heat load. The modification of the deposited heat load due to $\mathrm{N}$ and $\mathrm{D}$ fuelling has been studied using two independent diagnostics, namely, a set of thermocouples embedded into the divertor tiles (this system is very reliable and not perturbed by any carbon surface layer effect, but it measures only the bulk temperature) and the IR wide angle viewing system that measures the surface temperature [18].

The energy distribution in the divertor integrated all over the discharge, derived from the thermocouple (TC) measurement, shows a significant reduction in tile Nos 5 and 6 (where the OSP is located, as shown in figure 1, and where the inter-ELM heat load is maximal) when the N-fuelling rate is increased. The total energy loaded into the outer tile no 6 during the pulse is $\sim 25 \mathrm{MJ}$ without and $\sim 20 \mathrm{MJ}$ with $\mathrm{N}$ seeding, while it remains constant when increasing the deuterium fuelling rate. This effect is even more visible when looking directly at the thermocouples data during the plateau when the fuelling is applied. As a first approximation, the heat load averaged over transient and stationary phases can be qualitatively estimated by looking at the difference in temperature $\Delta T_{\mathrm{TC}}$ (in degree centigrade) between the end and the beginning of the seeded phase, measured by one of the embedded TC (at $1 \mathrm{~cm}$ from the surface of the tile). Note that this qualitative estimation of Q does not depend on any carbon surface layer on the top of the tile (while it is sometimes problematic for the interpretation of the IR data). In figure 7 we plot the difference in temperature $\Delta T_{\mathrm{TC}}$ measured in tile no 6 (near the OSP) divided by the total input power in order to study the variation of the heat load (mainly made up of the conducted power near the OSP) when $\mathrm{N}$ and D fuelling is applied. In contrast to $\mathrm{D}$ injection alone that is completely inefficient for mitigating the surface temperature, $\mathrm{N}$ injection results systematically (i.e. independently of the preset deuterium fuelling rate-see $\mathrm{N}$ scans in figure 7) in lower $\Delta T_{\mathrm{TC}} / P^{\text {tot }}$, which implies lower heat loads. This effect is not completely understood and requires further investigations. In the low radiated fraction region (when $f^{\mathrm{rad}}$ inter-ELM is $<35 \%$ ), the averaged heat load reduction obtained with $\mathrm{N}$ injection is about a factor of $2\left(\Delta T_{\mathrm{TC}} / P^{\text {tot }} \sim 2.7^{\circ} / \mathrm{MW}\right.$ obtained with $\left.\Phi_{\mathrm{N}}^{3}\right)$. In the high radiated fraction region (when $f^{\text {rad }}$ inter-ELM is $>35 \%$ ), the maximum temperature reduction obtained with 


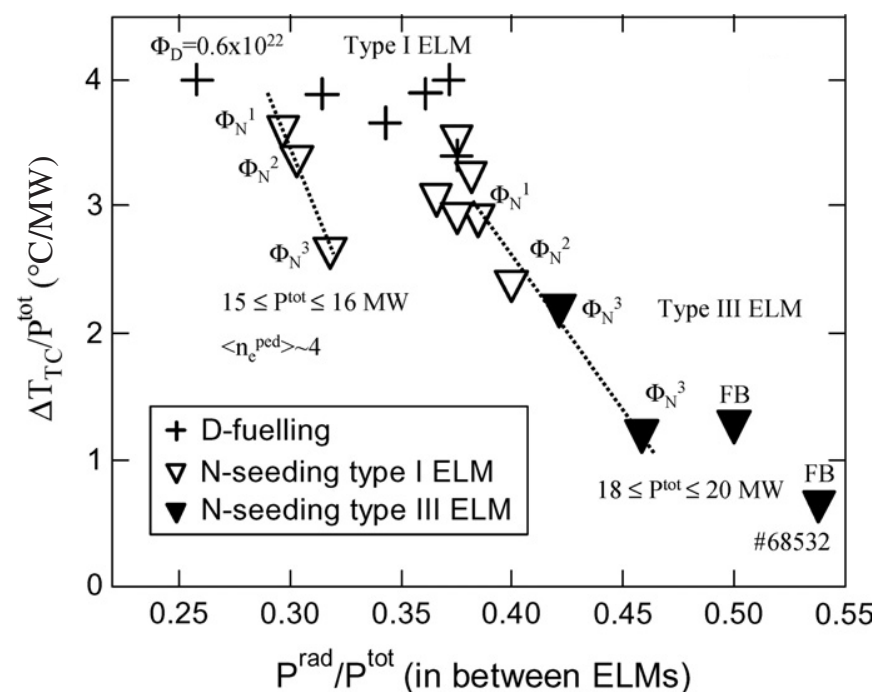

Figure 7. Difference of temperature measured in the bulk $\Delta T_{\mathrm{TC}}$ (outer tile no 6) during the $\mathrm{N}$-seeded phase divided by the total input power as a function of the radiated power fraction for a series of pulses with $\mathrm{D}$ (crosses) and $\mathrm{D}+\mathrm{N}$ (triangles) injection: $\Phi_{\mathrm{N}}^{1}=0.7 \times 10^{22} \mathrm{e} \mathrm{s}^{-1}$; $\Phi_{\mathrm{N}}^{2}=1.5 \times 10^{22} \mathrm{e} \mathrm{s}^{-1} ; \Phi_{\mathrm{N}}^{3}=2.2 \times 10^{22} \mathrm{e} \mathrm{s}^{-1}, \mathrm{FB}$ represents the radiative feedback control (with variable $\Phi_{\mathrm{N}}$ ).

nitrogen $\left(\Delta T_{\mathrm{TC}} / P^{\text {tot }} \sim 1.2^{\circ} / \mathrm{MW}\right.$ for $\left.\Phi_{\mathrm{N}}^{3}\right)$ compared with deuterium $\left(\Delta T_{\mathrm{TC}} / P^{\text {tot }} \sim 3.7^{\circ} / \mathrm{MW}\right.$ in average) injection is a factor of 3 near the OSP.

The benefit of $\mathrm{N}$ injection for reducing the heat loads is confirmed by the averaged (over transient and stationary phases during the seeding phase) peak heat flux estimated from the IR wide-angle viewing system [18]. On the OSP (tile no 6) the peak heat flux (maximum of the heat flux distribution computed along the surface of the poloidal cross section of the tiles with the 2D non-linear code THEODOR [19]) is reduced by almost a factor of 2 (therefore consistent with the TC data presented above): from $\left\langle Q_{\text {target }}^{\mathrm{OSP}}\right\rangle=1.7 \mathrm{MW} \mathrm{m}^{-2}$ with $\mathrm{D}$ fuelling only (reference pulse \#68505) to $\left\langle Q_{\text {target }}^{\text {OSP }}\right\rangle=0.9 \mathrm{MW} \mathrm{m}^{-2}$ with $\mathrm{N}$ fuelling $\left(\# 68510 ; \Phi_{\mathrm{N}}^{3}=2.2 \times 10^{22} \mathrm{e} \mathrm{s}^{-1}\right)$. This is also observed during a similar experiment using impurity seeding in the advance tokamak scenario with internal transport barrier (ITB) recently developed at JET [20,21]. $\mathrm{N}$ seeding alone enables to mitigate the energy and power load during the stationary phase keeping quasi-constant the pedestal temperature profile. To reduce the ELM energy, it is necessary to reach a higher radiative power fraction and hence get a degraded edge pedestal as reported in [22].

\section{Plasma modification observed during the hybrid type III ELM regime with $\mathrm{N}_{2}$ seeding}

\subsection{Edge pedestal}

The reference hybrid scenario with low D fuelling (\#68505: $\Phi_{\mathrm{D}}=0.6 \times 10^{22} \mathrm{e} \mathrm{s}^{-1}$ ) shows a pedestal ion temperature of $\sim 1000 \mathrm{eV}$ (measured at the top of the pedestal with the edge CXRS diagnostic [17] - figure 8). A slightly higher temperature is observed for the electron with the core and edge LIDAR Thomson scattering measurements [23] (figure 9 $(a)$ ).

Such plasma conditions are associated with type I ELM behaviour with a high peak heat flux (up to $\sim 60 \mathrm{MW} \mathrm{m}^{-2}$ as reported in JET $[20,24]$ ) leading to high erosion rates 
(a) averaged ion temperature profile $\mathrm{t}=[49-51 \mathrm{~s}]$

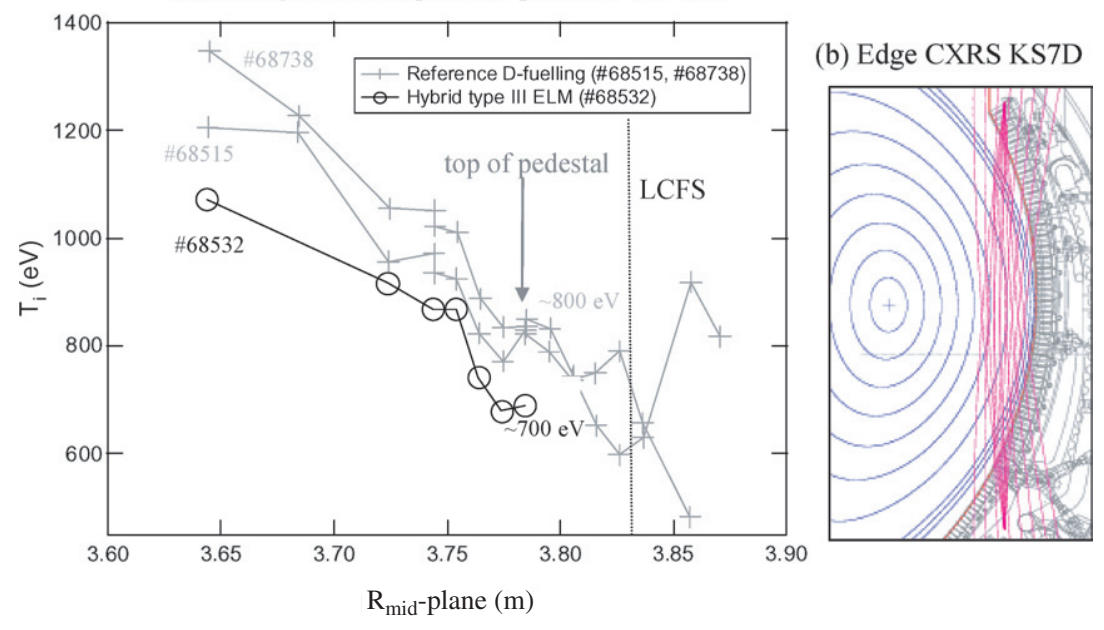

Figure 8. (a) Time average of the ion temperature profiles as measured with the edge charge exchange recombination spectroscopy (CXRS) diagnostic, averaged over $t=[8-11 \mathrm{~s}]$, for the reference hybrid high D fuelling (crosses: \#68515 and \#68738) and the hybrid type III ELM (circles: \#68532) discharges. (b) Lines of sight of the edge CXRS diagnostics in the JET poloidal section.

(a) averaged electron temperature profile

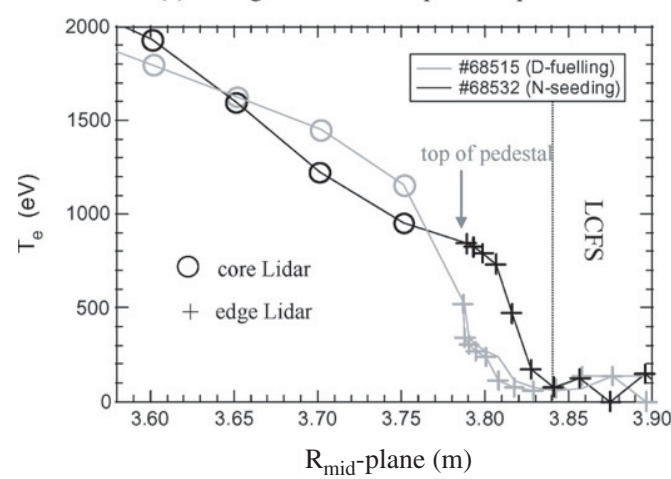

(b) averaged electron density profile

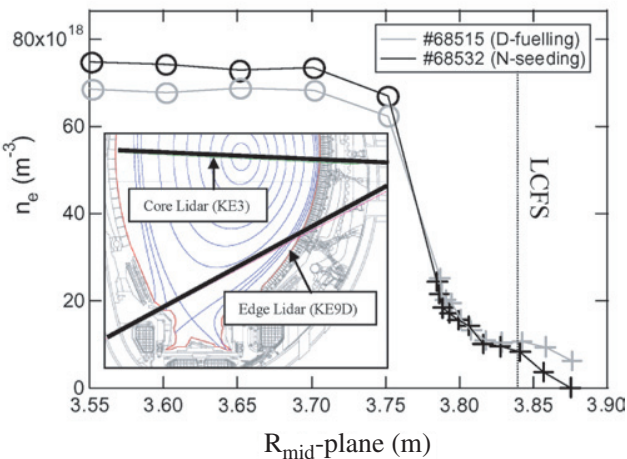

Figure 9. Electron $(a)$ temperature and $(b)$ density profiles such as measured with the core and edge LIDAR Thomson scattering diagnostics, time average over $t=[9-11 \mathrm{~s}]$. The lines of sight for the edge and core LIDAR diagnostics in the JET poloidal section are also indicated.

during ELMs. As discussed in the previous section, increasing deuterium fuelling (\#68515: $\Phi_{\mathrm{D}}=5 \times 10^{22} \mathrm{e} \mathrm{s}^{-1}$ ) reduces the pedestal ion temperature below $800 \mathrm{eV}$. The radiated fraction does not increase significantly (maximum $f^{\text {rad }}$ reached with D fuelling is $\sim 37 \%$, see figure $3(a)$, and the averaged ELM frequency stays around $50-65 \mathrm{~Hz}$, see table 1) and the type III ELMs regime is not achieved. For a higher level of $\mathrm{D}$ injection $\left(\Phi_{\mathrm{D}}=5 \times 10^{22} \mathrm{e} \mathrm{s}^{-1}\right)$ adding $\mathrm{N}$ seeding leads to a simultaneous increase in the radiated fraction up to $50 \%$, while the pedestal ion temperature drops below $750 \mathrm{eV}$ (figure 8(a)), and the type III ELM regime is triggered.

The edge plasma modifications (in the pedestal region) due to D-fuelling type I ELM and $\mathrm{N}$-fuelling type III ELM are also illustrated in figure 9. In addition to the observed cooling of the ions in the pedestal region, the temperature gradients are different with and without $\mathrm{N}$ seeding. 
(a) Radiated power (horizontal camera)

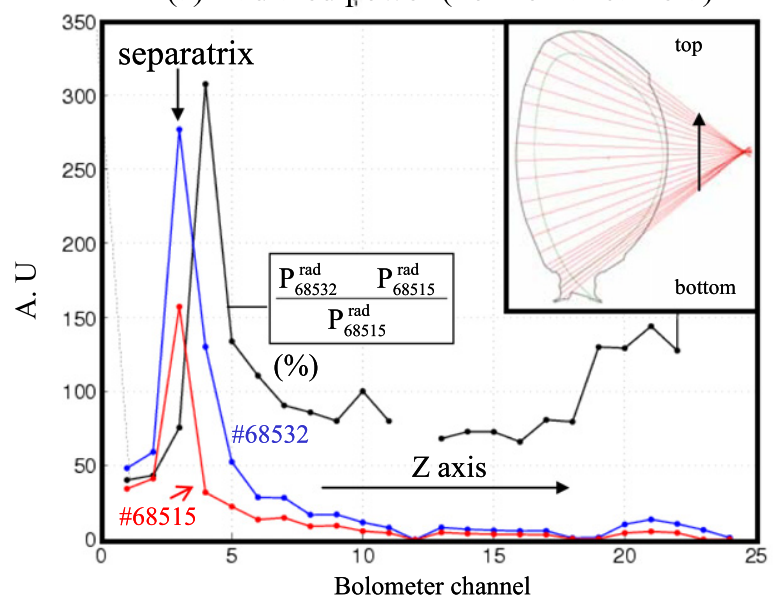

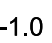

$-1.0$

$-1.2$

$-1.4$

$-1.6$

$-1.0$

$-1.2$

$-1.4$

$-1.6$

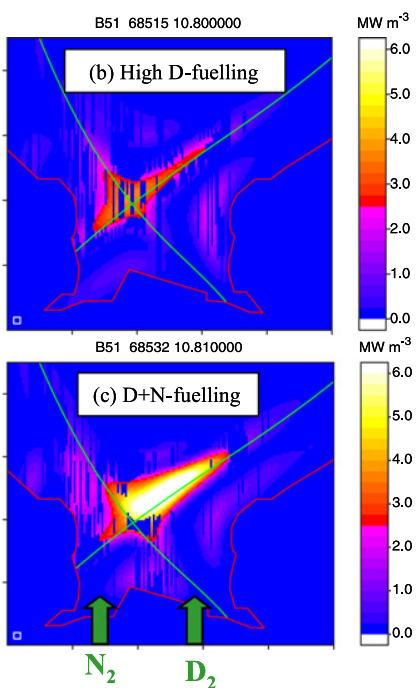

Figure 10. (a) Radiated power profile along the vertical axis taken in the inter-ELM phase, for the reference hybrid scenario (\#68515) and hybrid type III ELM scenario (\#68532). Radiated power modification due to $\mathrm{N}$ seeding in \% (black curve). Tomographic reconstructions of $P^{\mathrm{rad}}$ in the divertor: $(b)$ reference hybrid and $(c)$ hybrid type III ELM scenario with $\mathrm{N}$ seeding.

The electron temperature gradient (figure $9(a)$ ) is outboard-shifted (by about $3 \mathrm{~cm}$ ) when $\mathrm{N}_{2}$ is seeded while the electron density gradient does not move (figure $9(b)$ ). This modification is not yet explained. Because of a possible change shot to shot in the laser beam alignment of the edge LIDAR Thomson scattering diagnostic (leading to probable high uncertainties on the radial position), optimized edge plasma measurements (using, for example, the newly installed JET high resolution Thomson scattering system [25] that produces high spatial resolution profiles of $n_{\mathrm{e}}, T_{\mathrm{e}}$ and $p_{\mathrm{e}}$ ) will be necessary to better characterize and validate such behaviour.

\subsection{Plasma radiation}

As presented in figure $10(a)$, the modification of the plasma radiation due to $\mathrm{N}$ seeding is illustrated by the horizontal bolometer camera measurements taken during the inter-ELM phase (viewed from the bottom to the top of the machine). Thomographic reconstructions of $P_{\text {rad }}$ derived from the horizontal and vertical bolometer cameras are presented in figures $10(b)$ and $(c)$. The divertor radiation is increased by almost a factor of 2 near the X-point (bolometer channel no 3 as indicated in figure $10(a)$ ) and by about a factor of $3(300 \%)$ just inside the separatrix (bolometer channel no 4), traducing an increase in the radiated volume. The radiated power into the divertor region is estimated to be $P_{\text {rad }}^{\text {div }} \sim 4 \mathrm{MW}$ without (\#68515) and $P_{\text {rad }}^{\text {div }} \sim 7 \mathrm{MW}$ with $\mathrm{N}$ seeding (\#68532).

In addition, the signals measured by the lines of sight passing through the core (channel nos 5-20) are increased by almost a factor of 2 on average. This is consistent with the total bulk radiation that is estimated to be $P_{\text {rad }}^{\text {bulk }} \sim 2 \mathrm{MW}$ without and $P_{\text {rad }}^{\text {bulk }} \sim 4 \mathrm{MW}$ with type III ELM obtained with $\mathrm{N}$ seeding. The relatively high bulk radiation is due to impurity contamination, as suggested by the increase in $Z_{\text {eff }}$ (shown in figure 4) and the nitrogen core concentration that is measured with the core CXRS diagnostic: $\mathrm{N}^{7+} / n_{\mathrm{e}} \sim 2 \%$ (data shown later on, in figure 16). The volume of plasma mostly affected by the nitrogen radiation is near the X-point, preferably inside the separatrix as also illustrated in figures $10(b)$ and $(c)$. 


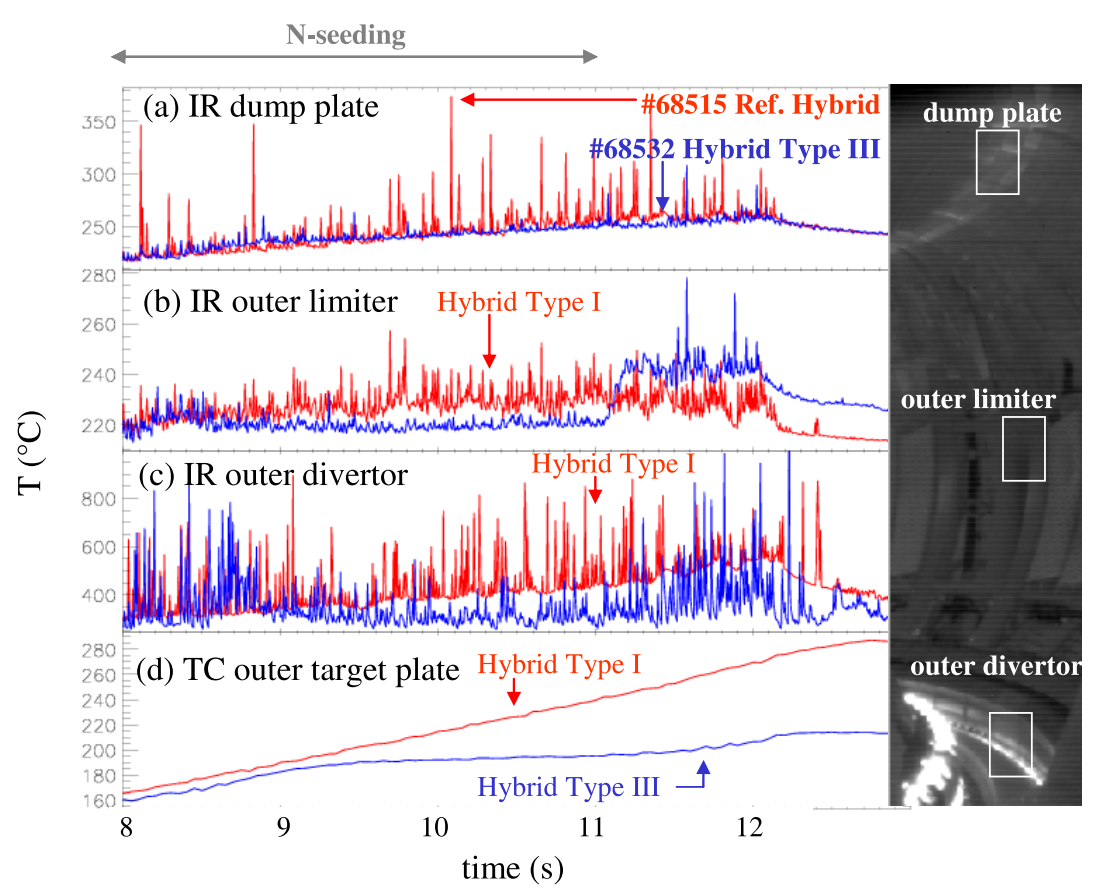

Figure 11. (a) Maximum surface temperature on the dump plate, $(b)$ the outer limiter and $(c)$ the outer divertor region. $(d)$ Bulk temperature into the horizontal target plate (at $1 \mathrm{~cm}$ deep) in the low-field side-below the OSP. N seeding is applied during $t=8-11 \mathrm{~s}$.

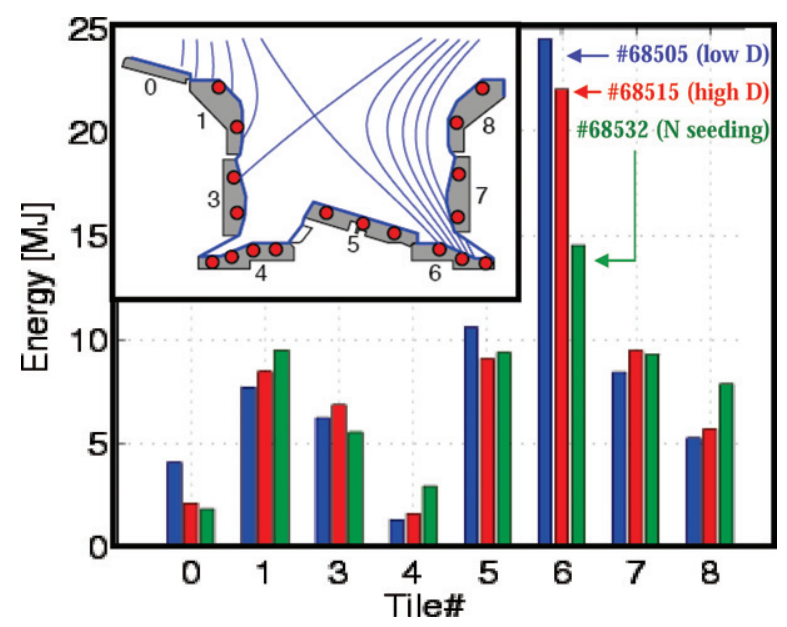

Figure 12. Energy distribution loaded in the divertor tiles (integrated over the all discharge) during reference and $\mathrm{N}$-seeding pulses.

\subsection{Heat load on targets}

The heat load reduction associated with the type III ELM regime is illustrated in figure 11 (IR data with a sampling rate of $6 \mathrm{~ms}$ ) and figure 12 (TC data with a sampling rate of $50 \mathrm{~ms}$ ). The 
amplitude of the surface temperature measured with the wide-angle thermographic viewing system [18] shows a net reduction during the $\mathrm{N}$-seeding phase from $t=8$ to $11 \mathrm{~s}$ (during transient and stationary phases of the discharge). During the inter-ELM periods, when the plasma is in quasi-stationary condition after the ELM collapse, the surface temperature remains stable ( $T^{\text {surf }}$ is controlled) on the outer target plate where the heat flux is expected to be maximum (blue curve in figure 11(c)). During ELMs, the IR sampling rate is not optimized to measure the true surface temperature (especially during the type III ELM regime where $\left\langle f_{\mathrm{ELM}}\right\rangle \sim 500 \mathrm{~Hz}$ ). The surface temperature variation $\Delta T_{\mathrm{ELM}}$ measured during ELMs can be used anyway, in first approximation, to study their amplitude. Figure 11 shows that $\Delta T_{\mathrm{ELM}}$ is almost completely mitigated $\left(\left\langle\Delta T_{\mathrm{ELM}}\right\rangle \sim 10^{\circ}\right)$ on the upper dump plate and outer limiter and partially reduced $\left(\left\langle\Delta T_{\mathrm{ELM}}\right\rangle \geqslant 200^{\circ}\right.$ during the type I and $\sim 100^{\circ}$ during the type III ELM regime) on the outer target plate region. Optimized IR data (with higher frame rate and reduced image size, up to $10 \mathrm{kHz}$ is achievable with the JET wide-angle thermographic system [26]) are necessary to quantify the ELM mitigation effect. Simultaneously, the bulk temperature is stabilized on the outer target plate where the heat flux is normally higher with forward magnetic field [27] ( $T^{\text {bulk }}$ is controlled, thus reinforcing the previous IR statement).

The total energy load on the outer tile no 6 over all the pulse is $15 \mathrm{MJ}$ with the hybrid type III ELM scenario and $25 \mathrm{MJ}$ during the reference hybrid type I ELM scenario (figure 12). The time-averaged (over transient and stationary phases during the seeding phase) peak heat flux computed on the OSP (with the 2D non-linear code THEODOR [19]) during the type III ELM hybrid regime is $\left\langle Q_{\text {target }}^{\text {OSP }}\right\rangle=0.4 \mathrm{MW} \mathrm{m}^{-2}$, corresponding to a net peak heat load reduction in both the ELM and inter-ELM periods of about a factor 4 compared with the reference hybrid type I ELM regime (where $\left\langle Q_{\text {target }}^{\text {OSP }}\right\rangle=1.7 \mathrm{MW} \mathrm{m}^{-2}$ ).

\subsection{Erosion on targets}

In addition to the deuterium (D) fuelling, the nitrogen $(\mathrm{N})$ seeding in the divertor can also modify the plasma-wall interaction and sputtering processes, which play a role in the erosion rate of the target plates and consequently in the PFC lifetime (a critical issue for the divertor target plate in ITER). The modification of molecular (CD and CN band) and carbon (CII line) emissions in the divertor due to the $\mathrm{N}$ fuelling is observed with two spectrometers (labelled $\mathrm{KT} 3 \mathrm{~A} / \mathrm{B}$ ) monitoring the outer strike point (OSP) region with a good spectral and spatial resolution $(12.5 \mathrm{~mm})[28,29]$. The first spectrometer $(\mathrm{KT} 3 \mathrm{~A})$ monitors the divertor targets in the near UV wavelength range $(\lambda=384-398 \mathrm{~nm})$ and allows the detection of CN, CII and NII emission (figure 13). The second spectrometer (KT3B) monitors the same region in the visible wavelength range ( $\lambda=426-429 \mathrm{~nm}$ ) and allows the detection of CD and CII emission. The time evolution of the released species due to $\mathrm{N}$ seeding is depicted in figure 14, showing a significant reduction in the CD Gerö band $\left(A^{2} \Delta \rightarrow X^{2} \Pi\right)$ and CII line emission during $\mathrm{N}$ seeding.

This can be explained in part by a reduction of the plasma density near the OSP, as measured by the Langmuir probe: $\left\langle n_{\mathrm{e}}^{\text {edge }}\right\rangle \sim 10^{19} \mathrm{~m}^{-3}$ during the reference hybrid pulse and $\left\langle n_{\mathrm{e}}^{\text {edge }}\right\rangle \sim 2 \times 10^{18} \mathrm{~m}^{-3}$ during the hybrid type III ELM pulse (suggesting a possible partial plasma detachment) while the edge plasma temperature stays constant ${ }^{1}\left(\left\langle T_{\mathrm{e}}^{\text {edge }}\right\rangle\right.$ lies between 5 and $10 \mathrm{eV}$ near the OSP during both the reference and the type III ELM hybrid regimes). Since no measurements precisely localized at the OSP are available, it is not clear whether a

1 Note that the Langmuir probe used in this section is not located exactly on the OSP but a little bit further away in the direction of the private flux region. 


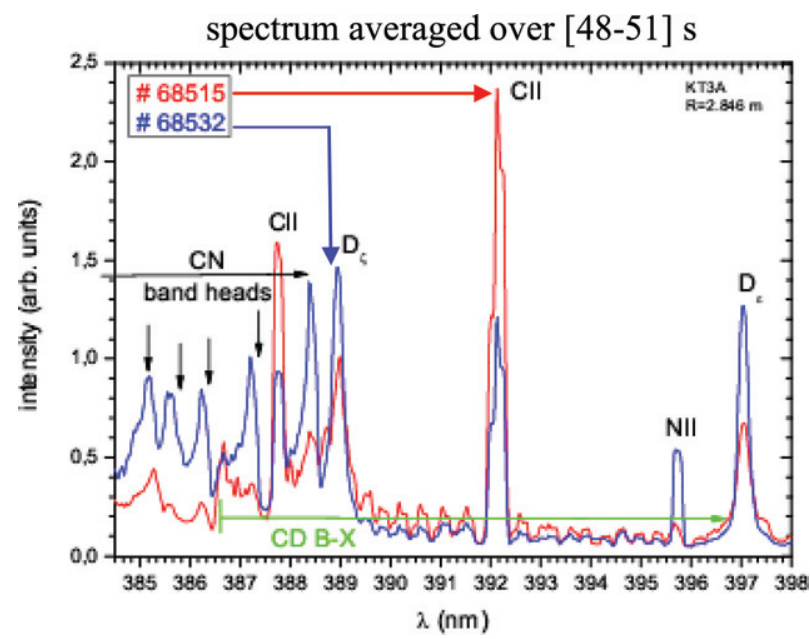

Figure 13. KT3A UV spectrum averaged over the N-seeding phase (therefore ELM and inter-ELM averaged) showing $\mathrm{CN}$ bands in the discharge \#68515 (reference) and \#68532 (N-seeded).
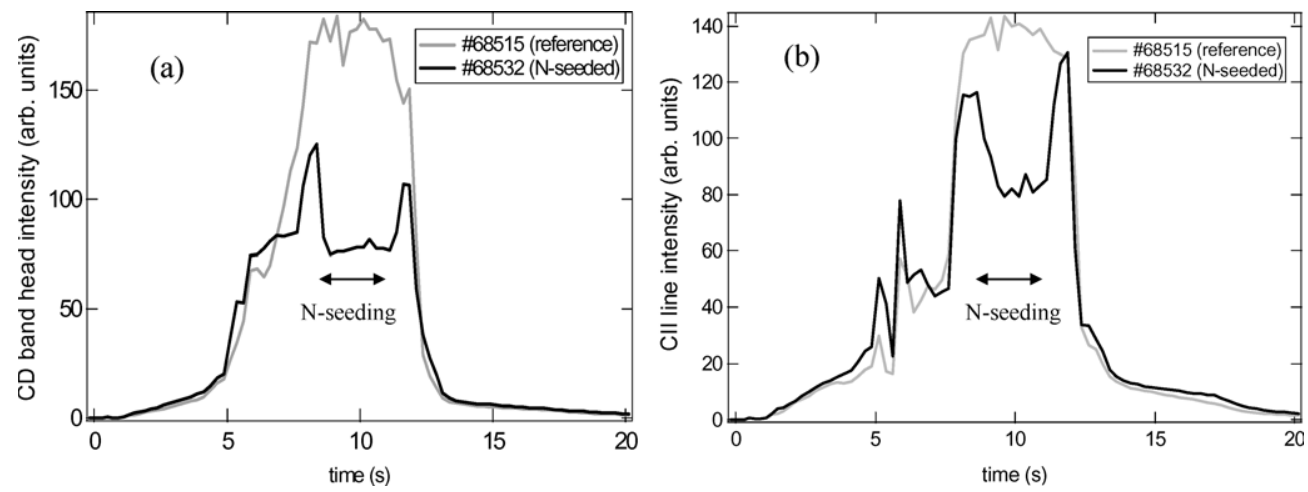

Figure 14. (a) Time evolution of the CD Gero band (integrated over the range $\lambda=429.5-431 \mathrm{~nm}$ ) and $(b) \mathrm{CII}(\lambda=426.7 \mathrm{~nm})$ line intensity during the reference (\#68515) and type III ELM (\#68532) hybrid discharges showing a reduction in $\mathrm{CD}$ and $\mathrm{CII}$ emission with $\mathrm{N}$ injection.

cooling effect is produced on the target or not. The variation of the plasma density modifies directly the line emission efficiency (proportional to the electronic density) but also indirectly (through the incident particle flux) the sputtering processes and thus the carbon sources. In the meantime, the $\mathrm{CN}$ molecule emission becomes higher (see the averaged spectrum over the seeding phase in figure 13) indicating an increase in the chemical processes involving nitrogen and carbon. Further investigations relying on particle flux and erosion yield calculation (the procedure is described in [30]) are required to determine which one of the following factors is responsible for the global reduction in CII: the 'density' effect alone or with an additional 'cooling' effect (leading to a lower sputtering yield) or the 'chemical' effect (due to a possible film suppression and a particular low erosion rate in the presence of active species such as $\mathrm{N}_{2}$ [31]). New plasma experiments with the OSP localized precisely on the Langmuir probe and quartz micro-balance data with at least $5 \mathrm{~s}$ exposure time (to get rid of the noise) to monitor the film growth or suppression [32] would be required. 


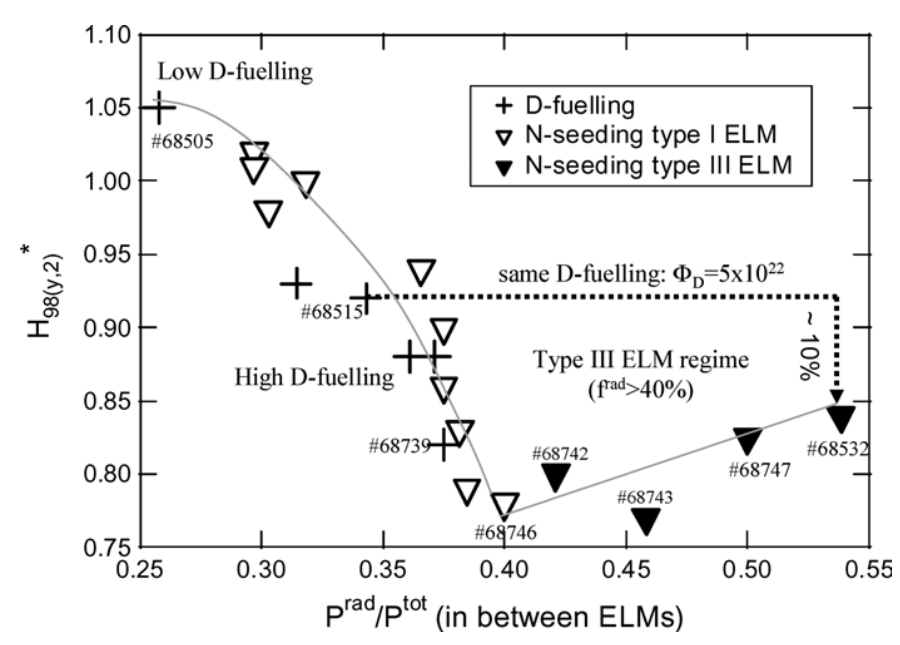

Figure 15. Thermal confinement enhancement factor $\mathrm{H} 98_{(y, 2)}^{*}$ versus the radiated fraction measured in between ELMs for a series of pulses with D fuelling only (crosses) and D $+\mathrm{N}$ fuelling (white and black triangles are associated with type I and III ELM regimes, respectively).

\section{Integrated hybrid type III ELM performance}

The hybrid type III ELM scenario obtained by $\mathrm{N}$ seeding has been successfully developed in JET with $I_{\mathrm{p}}=1.7 \mathrm{MA}, q_{95} \sim 3.2, n_{\mathrm{e}} \sim 0.95 \cdot n_{\mathrm{Gr}}$ and total $\beta_{\mathrm{N}} \sim 2.6$. The plasma performance of this scenario is illustrated in figure 15 , which represents the global energy confinement factor $\mathrm{H} 98_{(y, 2)}^{*}$ as a function of the radiated fraction for a series of hybrid discharges with D fuelling and a mixture of $\mathrm{D}+\mathrm{N}$ fuelling. The contribution of fast particles (corresponding on average to $\sim 15 \%$ reduction in the nominal confinement value) has been subtracted in order to identify the thermal part of the confinement. With pure deuterium fuelling, the standard $\mathrm{H}$-mode behaviour is observed: at low D fuelling (\#68505) the hybrid discharges achieve $\mathrm{H} 8_{(y, 2)}^{*} \geqslant 1$, while high D fuelling is accompanied by a reduction in the global confinement: $\sim 10 \%$ losses with $\Phi_{\mathrm{D}}=5 \times 10^{22} \mathrm{e} \mathrm{s}^{-1}$ (\#68515) and $\sim 20 \%$ losses with $\Phi_{\mathrm{D}}=9 \times 10^{22} \mathrm{e} \mathrm{s}^{-1}$ (\#68739) without significant increase in the radiated fraction. Although $\mathrm{N}$ seeding also reduces the global energy confinement, it enables a higher radiated fraction to be reached than with $\mathrm{D}$ fuelling alone. The degradation of global confinement associated with the type III ELM regime is about $10 \%$ compared with the reference hybrid high D-fuelling discharge (\#68515), which uses the same D fuelling: $\Phi_{\mathrm{D}}=5 \times 10^{22} \mathrm{e} \mathrm{s}^{-1}$. The observed loss of energy confinement has to be balanced with the reduction in the peak heat flux by a factor of $4:\left\langle Q_{\text {target }}^{\text {OSP }}\right\rangle=1.7 \mathrm{MW} \mathrm{m}^{-2}$ is measured during the reference high D-fuelling hybrid regime \#68515 and $\left\langle Q_{\text {target }}^{\text {OSP }}\right\rangle=0.4 \mathrm{MW} \mathrm{m}^{-2}$ during the type III ELM hybrid regime \#68532.

Figure 15 shows that the confinement is stabilized or even slightly improved when the radiative fraction is high $\left(\mathrm{H}_{9} 8_{(y, 2)}^{*}\right.$ lies between 0.75 and 0.83 when $\left.P^{\text {rad }} / P^{\text {tot }} \geqslant 0.4\right)$. A first series of possible candidates responsible for the stabilization of the $\mathrm{H} 8_{(y, 2)}^{*}$ factor observed in the newly developed type III ELM regime could be

- the quantity of nitrogen already present in the machine (trapped and released from the previous discharges),

- the amplitude of the $\mathrm{N}$-fuelling rates which are different,

- different MHD properties (but the similar MHD characteristics observed in this series of discharges seem to discredit this cause), 


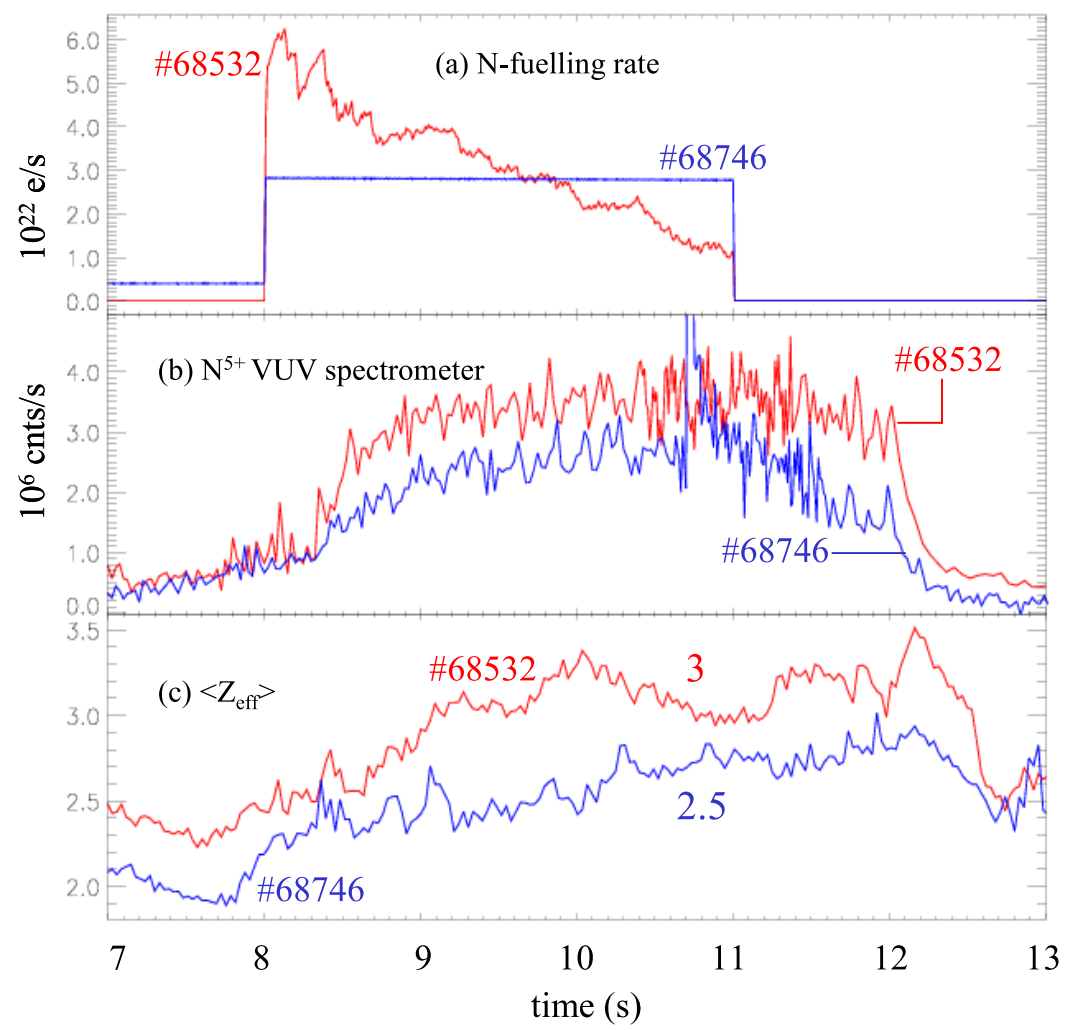

Figure 16. (a) N-fuelling rate (HFS). (b) $\mathrm{N}^{5+}$ radiation measured with the VUV spectrometer. (c) $Z_{\text {eff }}$ measured during pulses \#68532 and \#68746.

- the possible sensitivity of the nitrogen transport (penetration and dilution into the main plasma) to the plasma edge characteristics (position, amplitude and stiffness of the pedestal, temperature and density gradients).

This is illustrated in figures 16 and 17 when comparing pulse \#68746 (pre-programmed and lower $\mathrm{N}$-fuelling rate) with pulse \#68532 (feedback and highest $\mathrm{N}$-fuelling rate). Using a similar and constant $\mathrm{D}$-fuelling rate, the latter has about $10 \%$ better core performances.

The pulse with the lower $\mathrm{N}$-fuelling rate at the beginning of the plateau (\#68746, which also has lower $\mathrm{N}^{5+}$ brightness as shown in figure $16(b)$ ) ends up with a mixed phase of small type I and type III ELM phases while the pulse with the highest $\mathrm{N}$-fuelling rate at the beginning ends up with a pure type III ELM behaviour, a high $Z_{\text {eff }}(\sim 3)$ and $P^{\text {rad }} / P^{\text {tot }}>40 \%$. In the plasma core, the nitrogen concentration (measured with the core charge exchange recombination spectroscopy system $[33,34])$ in the high $\mathrm{N}$-fuelling case $\left(\mathrm{N}^{7+} / n_{\mathrm{e}} \sim 2 \%\right)$ is about twice the value measured in the moderate and constant $\mathrm{N}$-fuelling case $\left(\mathrm{N}^{7+} / n_{\mathrm{e}} \sim 1 \%\right)$-as shown in figure $17(a)$.

In the plasma core, impurity seeding does not systematically cool the plasma because of radiation. In contrast to the plasma edge, the plasma core contains low- $Z$ impurities that are mostly completely striped and the radiated power becomes negligible compared with the convected-conducted power. In this region, the increase in impurity concentration (and $Z_{\text {eff }}$ ) can have various effects on the plasma properties. It can have effects on the plasma resistivity 
(a) core CXRS

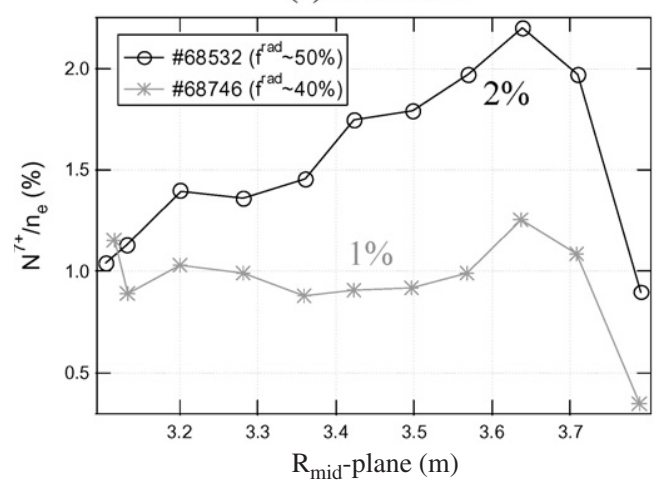

(b) core Lidar

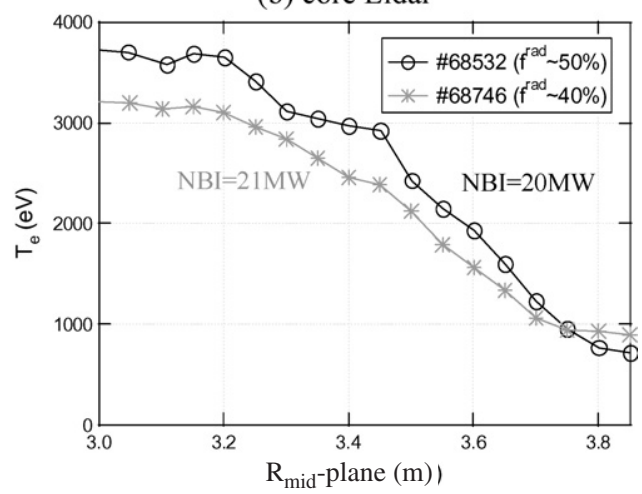

Figure 17. (a) N concentration and (b) electron temperature in the plasma core averaged over the seeding phase ( $t=48-51 \mathrm{~s}$ ) measured during pulses \#68532 (high start-up N-fuelling rate) and \#68746 (moderate start-up N-fuelling rate).

( $\propto$ impurity concentration), on the power absorption ( $\propto$ density) and also on the transport and confinement properties (driven by instabilities and anomalous transport). Figure $16(b)$ shows that the central electron temperature does increase when the $\mathrm{N}$ concentration (measured with the core charge exchange recombination spectroscopy system $[33,34]$ and plotted in figure $17(a)$ ) and $Z_{\text {eff }}$ increase: $T_{\mathrm{e}}^{\text {central }}=3200 \rightarrow 3700 \mathrm{eV}$ whereas $Z_{\text {eff }}=2.5 \rightarrow 3$. One possible mechanism responsible for the increase in the central temperature in impurity seeded plasmas is the suppression of the ion temperature gradient (ITG) instability through the increase in the ion effective charge [35]. A complete and dedicated transport (using integrated modelling codes such as TRANSP or CRONOS [36]) and stability analysis (using stability codes such as KINEZERO [37]) is required to validate this hypothesis and check if the increase in the central temperature when $Z_{\text {eff }}$ is higher could be responsible for the relative improvement in

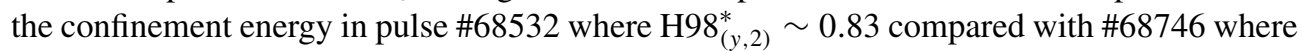
$\mathrm{H} 98_{(y, 2)}^{*} \sim 0.77$.

\section{Conclusions}

The type III ELM regime has been achieved during hybrid H-mode operation in the JET tokamak (with $I_{\mathrm{p}}=1.7 \mathrm{MA}, B_{\mathrm{t}}=1.7 \mathrm{~T}, n_{\mathrm{e}} \sim 0.95 \cdot n_{\mathrm{Gr}}$ and $\beta_{\mathrm{N}} \sim 2.6$ ) using both deuterium and nitrogen fuelling (this regime is triggered here when the inter-ELM radiated fraction reaches approximately $40 \%$ ). The newly developed integrated hybrid type III ELM scenario shows acceptable edge plasma conditions for the wall and moderate MHD activity including mild $n=1$ sawteeth precursors and residual MHD island (4/3 NTM), which means $q_{\text {min }}$ is close to unity. The core $q$-profile of the integrated hybrid type III ELM is very similar to the $q$-profile of the reference hybrid type I ELM scenario, indicating that it is also compatible with high $\beta_{\mathrm{N}}$ operation with minimized impact of sawteeth on stability (which leads, for example, to lowering the probability of triggering $3 / 2$ and $2 / 1$ NTMs). The target temperature on the OSP is significantly reduced and the peak heat flux is reduced by about a factor of 4 compared with the reference hybrid type I ELM scenario. The carbon erosion is also reduced and partially replaced by nitrogen chemical activity. The counterpart effect of the improvement in the edge conditions (concerning the plasma-wall interaction issue) is a reduction of $\sim 20 \%$ of the total plasma energy confinement $\left(\mathrm{H} 98_{(y, 2)}^{*} \sim 0.83\right.$ is obtained during the hybrid type III ELM 
scenario). This situation is identical to the case of the 'standard $\mathrm{H}$ mode' with large sawteeth: the transition from type I to type III ELMs also causes $\sim 20 \%$ degradation of the energy confinement time. A marginal but interesting plasma core effect, depicted by the relative increase in the central plasma temperature when the effective charge of the plasma increases, has been observed in the type III ELM regime. This phenomenon is marginal for the plasma energy confinement but becomes essential for the comprehension of the core plasma behaviour when impurity seeding is applied.

Due to the low confinement achieved during the hybrid type III ELM regime $\left(\mathrm{H} 98_{(y, 2)}^{*} \sim\right.$ $0.83)$, the scenario proposed in this paper is presently not favorable for ITER operation with nominal fusion performance (pulsed operation with $Q=10$ and $\mathrm{H}_{98}^{*}(y, 2)=1$ ). A second physics objective of ITER is to meet $Q=5$ in steady state plasma operation. If the confinement time of future ITER hybrid discharges (presently foreseen at low plasma current $I_{\mathrm{p}} \sim 14 \mathrm{MA}$ ) is high enough to allow type III ELM operation with acceptable fusion performance $(Q>5)$, then the experimental procedure described here can be envisaged to control the edge plasma conditions and get sustainable heat load (compatible with the ITER walls) without modifying the core $q$-profile and thus the high $\beta_{\mathrm{N}}$ capability of the hybrid scenario (optimized for current drive sources and non-inductive current bootstrap).

In addition to the problem of confinement, the relatively high impurity content and the extrapolation to ITER remain important issues to demonstrate the viability of the hybrid type III ELM scenario as an integrated scenario for ITER. Promising techniques are currently used or under development to suppress the impurity accumulation, for example, injection of radio frequency power [38-40] or high densities operation with lower plasma core pollution [41]. A step before ITER operation, the newly developed hybrid type III ELM scenario, can also be used in JET for high $\beta_{\mathrm{N}}$ operation with the future ITER-like wall (with tungsten and beryllium inertial tiles, e.g. with no actively cooled system). Better understanding of the recycling properties of nitrogen, use of the real-time control, maximization of confinement as observed in ASDEX Upgrade [42] and impurity decontamination techniques will be essential to improve the performances and the reliability of the scenario.

\section{Acknowledgments}

This work, supported by the European Communities under the contract of Association between EURATOM and CEA, was carried out within the framework of the European Fusion Development Agreement. The views and opinions expressed herein do not necessarily reflect those of the European Commission

\section{(C) Euratom 2008.}

\section{References}

[1] ITER Physics Basis 1999 Nucl. Fusion 392137

[2] Sips A C C et al 2002 Plasma Phys. Control. Fusion 44 B69-83

[3] Luce T C et al 2003 Nucl. Fusion 43 321-9

[4] Joffrin E et al 2007 Nucl. Fusion 647 1664-72

[5] Joffrin E et al and JET-EFDA Contributors to the Work Programme 2005 Nucl. Fusion 45 626-34

[6] Matthews G F et al and ITER-Like Wall Team 2008 Development of divertor Tungsten coatings for the ITER-like wall project 18 th PSI Conf. (Toledo, Spain)

[7] Riccardo V 2008 on behalf of the ITER-Like Wall Engineering Design and Manufacturing Team. Engineering challenges of the JET ITER-like wall 18th PSI Conf. (Toledo, Spain)

[8] Rapp J et al 2005 J. Nucl. Mater. 337-339 826-30 
[9] Monier-Garbet P et al and JET-EFDA Contributors 2005 Nucl. Fusion 45 1404-10

[10] Saibene G et al 1999 Nucl. Fusion 39 1133-56

[11] Chankin A V and Saibene G 1999 Plasma Phys. Control. Fusion 41 913-30

[12] Sartori R et al 2004 Plasma Phys. Control. Fusion 46 723-50

[13] Joffrin E et al and Contributors to the JET EFDA Programme 2003 Plasma Phys. Control. Fusion 45 A367-83

[14] Petrie T W et al and DIII-D Team 2007 J. Nucl. Mater. 363-365 416-20

[15] Koslowski H R, Nave M F, Alper B, Perez von Thun C, Pinches S, Sauter O and Sharapov S E 200128 th EPS Conf. (18-22 June 2001, Funchal) vol 25A (ECA) pp 965-8

[16] Chang Z and Callen J D 1990 Nucl. Fusion 2219

[17] Andrew Y, Hawkes N C and Crombe K 2006 Rev. Sci. Intrum. 77 10E913

[18] Gauthier E, Andrew P, Arnoux G, Corre Y, Roche H and JET-EFDA Contributors 2007 J. Nucl. Mater. 363-365 1026-31

[19] Herrmann A, Junker W, Gunther K, Bosch S, Kaufmann M, Neuhauser J, Pautasso G, Richter Th and Schneider R 1995 Plasma Phys. Control. Fusion 37 17-29

[20] Beurskens M et al 2008 Nucl. Fusion 48095004

[21] Arnoux G et al and JET EFDA contributors 2008 18th PSI Conf. (Toledo, Spain)

[22] Rapp J, Eich T, von Hellermann M, Herrmann A, Ingesson L C, Jachmich S, Matthews G F, Philipps V, Saibene G and contributor to the EFDA-JET Workprogramme 2002 Plasma Phys. Control. Fusion 44 639-52

[23] Salzmann H, Bundgaard J, Gadd A, Gowers C, Hansen K B, Hirsch K, Nielsen P, Reed K, Schrödter C and Weisberg K 1988 The LIDAR Thomson scattering diagnostic on JET Rev. Sci. Instrum. 59 1451-6 (invited)

[24] Loarte A, Saibene G and Sartori R 2004 Phys. Plasmas. 11 2668-78

[25] Pasqualotto R, Nielsen P, Gowers C, Beurskens M, Kempenaars M, Carlstrom M and Johnson D 2004 Rev. Sci. Instrum. 753891

[26] Gauthier E et al 2007 Fusion Eng. Des. 82 1335-40

[27] Pitts R A et al 2005 J. Nucl. Mater. 337-339 146-53

[28] Stamp M F, Erents S K, Fundamenski W, Matthews G F and Monk R D 2001 J. Nucl. Mater. 290-293 321-5

[29] Brezinsek S, Pospieszczyk A, Stamp M F, Meigs A, Kirschner A, Huber A, Mertens Ph and JET-EFDA Contributors $2005 \mathrm{~J}$. Nucl. Mater 337-339 1058-63

[30] Brezinsek S et al 2005 32nd EPS Conf. (Tarragona, Spain)

[31] Tabarés F L, Rohde V and the ASDEX Upgrade Team 2004 Plasma Phys. Control. Fusion 46 381-95

[32] Esser H G, Kreter A, Philipps V, Widdowson A M, Coad J P and Stamp M 2008 18th PSI Conf. (Toledo, Spain)

[33] Negus C R, Giroud C, Meigs A G, Zastrow K-D and Hillis D L 2006 Rev. Sci. Instrum. $7710 \mathrm{~F} 102$

[34] Giroud C et al 2008 High Temperature Plasma Diagnostic Conf. (Albuquerque, New Mexico)

[35] Tokar M Z 2003 Plasma Phys. Control. Fusion 45 1323-32

[36] Basiuk V et al 2003 Nucl. Fusion 43 822-30

[37] Bourdelle C, Garbet X, Hoang T G, Ongena J and Budny R V 2002 Nucl. Fusion $42892-902$

[38] Dux R, Giroud C, Neu R, Peeters A G, Stober J and Zastrow K D 2003 J. Nucl. Mater. 313-316 1150-5

[39] Carraro L et al 2007 34th EPS Conf. (Warsaw, Poland)

[40] Nave M F F et al 2003 Nucl. Fusion 43 1204-13

[41] Rapp J et al 2008 18th PSI Conf. (Toledo, Spain)

[42] Sips ACCC et al 2002 Plasma Phys. Control. Fusion 44 A151 